\title{
Glucose-6-phosphate-dependent phosphoryl flow through the Uhp two-component regulatory system
}

\author{
Daniël T. Verhamme, Jos C. Arents, Pieter W. Postma, Wim Crielaard \\ and Klaas J. Hellingwerf
}

Swammerdam Institute for Life Sciences, University of Amsterdam, Nieuwe Achtergracht 166 , 1018 WV Amsterdam, The Netherlands
Author for correspondence: Klaas J. Hellingwerf. Tel: +31 20 5257055. Fax: +31 205257056. e-mail : K.Hellingwerf@chem.uva.nl

\begin{abstract}
Expression of the UhpT sugar-phosphate transporter in Escherichia coli is regulated at the transcriptional level via the UhpABC signalling cascade. Sensing of extracellular glucose 6-phosphate (G6P), by membrane-bound UhpC, modulates a second membrane-bound protein, UhpB, resulting in autophosphorylation of a conserved histidine residue in the cytoplasmic (transmitter) domain of the latter. Subsequently, this phosphoryl group is transferred to a conserved aspartate residue in the response-regulator UhpA, which then initiates uhpT transcription, via binding to the uhpT promoter region. This study demonstrates the hypothesized transmembrane signal transfer in an ISO membrane set-up, i.e. in a suspension of UhpBC-enriched membrane vesicles, UhpB autophosphorylation is stimulated, in the presence of $\left[\gamma_{-32}{ }^{32}\right.$ P]ATP, upon intra-vesicular sensing of G6P by UhpC. Subsequently, upon addition of UhpA, very rapid and transient UhpA phosphorylation takes place. When $P \sim$ UhpA is added to G6P-induced UhpBC-enriched membrane vesicles, rapid UhpA dephosphorylation occurs. So, in the G6P-activated state, UhpB phosphatase activity dominates over kinase activity, even in the presence of saturating amounts of G6P. This may imply that maximal in vivo $P \sim$ UhpA levels are low and/or that, to keep sufficient $P \sim$ UhpA accumulated to induce uhpT transcription, the uhpT promoter DNA itself is involved in stabilization/sequestration of $\mathbf{P} \sim$ UhpA.
\end{abstract}

Keywords: transmembrane signalling, kinase/phosphatase state

\section{INTRODUCTION}

Every living cell has to cope with environmental fluctuations in nutrient concentration and/or the stringency of 'stress' signals from the environment. Unicellular organisms such as bacteria have to be especially well equipped to respond appropriately to their (continuously) changing surroundings. To sense, transduce and correctly respond to various signals, prokaryotes predominantly rely on the intracellular covalent modification of sensory and regulatory proteins via transient phosphorylation of specific histidine and aspartate residues (Parkinson \& Kofoid, 1992; Perraud et al., 1999; Stock et al., 1989). The two prerequisite components in such a system are a sensory histidine-protein kinase (HPK) and its cognate response-regulator (RR).

Abbreviations: G6P, glucose 6-phosphate; HPK, histidine-protein kinase ; ISO, inside-out; RR, response-regulator; RSO, right-side-out.
Specificity resides in the input domain, triggering autophosphorylation on a conserved histidine residue in the transmitter domain of the HPK, upon receiving the proper stimulus. The high-energy phosphoryl group is subsequently transferred to a conserved aspartate residue in the receiver domain of the RR. The resulting molecular switch activates an output domain of the RR, which in most cases modulates transcription of target gene(s) by (enhanced) binding to specific sequences in their promoter region. The phosphorylated state of RRs is transient, due to intrinsic instability of the phospho-aspartate bond (autophosphatase activity) as well as phosphatase activity in the HPK transmitter domain or in additional regulatory protein(s). Jointly, the HPKs and RRs belong to the family of so-called two-component regulatory proteins, which can be clustered on the basis of their highly conserved transmitter and receiver domains (e.g. Ronson et al., 1987). Because of their abundance and multi-regulatory involvement in 
bacterial signal transduction, the major role of His-Asp phosphoryl transfer, based on the two-component paradigm, is beyond doubt. Generally, the more systems a bacterium possesses [from a few in Haemophilus influenzae and Helicobacter pylori (Grebe \& Stock, 1999 ) to around 60 in Pseudomonas aeruginosa (Rodrigue et al., 2000) and Caulobacter crescentus (Nierman et al., 2001)], the more flexible a lifestyle it can adopt.

In Escherichia coli, $29 \mathrm{HPK} / \mathrm{RR}$ pairs have been identified (Mizuno, 1997). One of these is the UhpB-UhpA couple (Weston \& Kadner, 1988). UhpB is a membrane-bound sensor kinase, with the notable feature that it contains eight putative transmembrane helices (Island et al., 1992) instead of four for the KdpD sensor (Zimmann et al., 1995) and two for the other functionally characterized membrane-bound HPKs in $E$. coli (Williams \& Stewart, 1999). Furthermore, a second intrinsic membrane protein, $\mathrm{UhpC}$, is required to sense the extracellular stimulus for this system, the presence of glucose 6-phosphate (G6P) in the periplasm. Subsequently, UhpC activates UhpB, presumably by inducing a conformational change in the latter (Island \& Kadner, 1993; Island et al., 1992; Weston \& Kadner, 1988; Wright et al., 2000). This uncommon form of transmembrane signalling then activates the typical phosphoryl transfer reactions inherent to a two-component signal transduction pathway. The cytoplasmic part of UhpB contains the characteristic HPK/ transmitter sequence motifs, i.e. the H-box, as well as the $\mathrm{N}$ - and G-box in the ATP-binding kinase domain (for a recent comparison see Kim \& Forst, 2001). Autophosphorylation of UhpB on the conserved histidine (His-313) may occur in a (homo)dimeric structure, with one kinase monomer catalysing phosphorylation of the other (i.e. in trans), as has been shown for other HPKs (Dutta et al., 1999; Ninfa et al., 1993). The RR UhpA, which is composed of a receiver domain that belongs to the RC1 subfamily of receiver domains (Grebe \& Stock, 1999) and an output domain belonging to the FixJ/NarL subfamily (Parkinson \& Kofoid, 1992), becomes activated by phosphorylation of Asp-54. Phosphorylated UhpA exhibits enhanced affinity for specific target sequences in the $u h p T$ promoter region (Dahl et al., 1997; Merkel et al., 1992), through which it can stimulate UhpT expression. The UhpT transporter enables E. coli to take up a broad range of organophosphate compounds from its environment, which can be used as carbon and energy source (Dietz, 1976). Nevertheless, UhpT expression is exclusively induced by extracellular G6P (Winkler, 1970), because of the specificity of UhpC (Goldenbaum \& Farmer, 1980).

The $u h p A B C T$ operon has been extensively studied for more than 30 years, with regard to its characteristics of induction, regulation of $u h p T$ transcription by UhpA, UhpB and UhpC, and specificity and kinetics of transport by UhpT (see Kadner, 1995, for a review). Recently, the interaction of the transmitter domain of UhpB with UhpA has been described in detail (Wright \& Kadner, 2001; Wright et al., 2000). Here we demonstrate the
G6P-induced transmembrane signal transfer through the UhpBC complex in an in vitro system, which results in UhpB autophosphorylation and subsequent phosphoryl transfer to UhpA. We have observed that G6P increases the kinase/phosphatase ratio in the UhpBC complex, thereby generating phosphorylated UhpA. Nevertheless, G6P does not seem to suppress the 'default' phosphatase-on state of UhpB.

\section{METHODS}

Plasmid construction. DNA corresponding to the $u h p A$ ORF was amplified by PCR with primers UHPA1 (5'-GCGGATCCGATGACGATGACAAAATGATCACCGTTGCCCTTATAG-3') and UHPA2 (5'-GCGCAAGCTTCACCAGCCATCAAACATGC-3'). DNA encoding the cytoplasmic portion of $u h p B$ was amplified by PCR with primers UHPB1 (5'-GCGGATCCCTGGCTGAACGGTTGCTGG-3') and UHPB2 (5'-GCGCAAGCTTAGACATAGCGTTGAGGTAG-3'). Pwo DNA polymerase (Boehringer Mannheim) was used, with E. coli MC4100 [ $\Delta$ (argF-lac)U169 araD139 rpsL150 relA1 flbB5103 deoC1 ptsF25 rbsR22; Silhavy, 1984] chromosomal DNA as a template. PCR products were digested with Bam HI and HindIII and cloned into the corresponding sites of pQE30 (Qiagen), resulting in expression vectors for full-length UhpA (pQE30uhpA) and a truncated form of UhpB (residues 293-500, pQE30uhpB'). Both recombinant proteins (i.e. UhpA and $\mathrm{UhpB}^{\prime}$ ) were produced with an amino-terminal $\mathrm{His}_{6}{ }^{-}$ extension, under control of a phage $\mathrm{T} 5$ promoter regulated by IPTG (for further details see Qiagen brochure). The cloned $u h p A$ and $u h p B^{\prime}$ fragments were confirmed by DNA-sequence analysis, using an ABI system (sequence facility, Free University, Amsterdam).

Purification of $\mathrm{His}_{6}-\mathrm{UhpA}$ and $\mathrm{His}_{6}-\mathrm{UhpB}^{\prime}$. $\mathrm{His}_{6}$-tagged recombinant proteins were isolated from E. coli M15 cells (Qiagen), transformed with pREP4 (Qiagen) and either

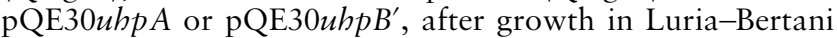
medium (Sambrook et al., 1989) at $37^{\circ} \mathrm{C}$ and induction with IPTG $(1 \mathrm{mM})$. The proteins were purified by nickel chelate affinity chromatography using a Ni-NTA resin (Qiagen), according to the QIAexpressionist manual (Qiagen). Purification under non-denaturing conditions resulted in a highly concentrated, soluble $\mathrm{His}_{6}$-UhpA fraction, whereas overproduced $\mathrm{His}_{6}-\mathrm{UhpB}^{\prime}$ largely ended up in the insoluble fraction, due to the formation of inclusion bodies. $\mathrm{His}_{6}{ }^{-\mathrm{UhpB}^{\prime}}$ was purified from this fraction under denaturing conditions with $8 \mathrm{M}$ urea. The resulting (dialysed) product was subsequently only used to raise antiserum. Final protein preparations were $\geqslant 95 \%$ pure, as analysed on SDS-PAGE. Protein concentrations were determined using the Bradford assay (Bio-Rad) with BSA as a standard. Proteins were stored in small aliquots at $-20{ }^{\circ} \mathrm{C}$.

Isolation of inside-out membrane (ISO) vesicles. For preparation of UhpBC-enriched- and UhpBC-lacking- (i.e.' empty') membrane vesicles, E. coli RK1448 transformed with plasmid pRK18 $\left[u h p(\Delta A)(B C)^{+}(\Delta T)\right.$ in pBR322] and E. coli RK5000 $(\triangle u h p A B C T)$ were used, respectively. Strains and plasmid (Weston \& Kadner, 1988) were kindly provided by R. J. Kadner (University of Virginia, Charlottesville, USA). Cell cultures were grown aerobically in Luria-Bertani medium at $37^{\circ} \mathrm{C}$ to an $\mathrm{OD}_{600}$ of $\sim 3$. Cells were harvested by centrifugation $(20 \mathrm{~min}$ at $5000 \mathrm{~g})$ at $4{ }^{\circ} \mathrm{C}$, washed once with ice-cold buffer A (100 mM Tris/ $\mathrm{HCl} \mathrm{pH} \mathrm{7.4;} 5$ mM EDTA; $10 \%$, v/v, glycerol), and resuspended in 0.01 vol. buffer A with $1 \mathrm{mM}$ PMSF. The cell suspension was passed through a French 
pressure cell twice (12000 p.s.i.; 82.8 MPa). Cell debris was subsequently removed by centrifugation (twice, $20 \mathrm{~min}$ at $20000 \mathrm{~g}$ ). The resulting supernatant was centrifuged at $180000 \mathrm{~g}$ for $90 \mathrm{~min}$, to collect the membrane fraction. This membrane pellet was washed and resuspended in buffer $\mathrm{B}$ (50 mM HEPES $\mathrm{pH} 7 \cdot 8 ; 2 \mathrm{M} \mathrm{KCl} ; 1 \mathrm{mM}$ EDTA; $10 \mathrm{mM} \beta$ mercaptoethanol; $1 \mathrm{mM}$ PMSF; $10 \%, \mathrm{v} / \mathrm{v}$, glycerol) and sedimented again $(45 \mathrm{~min}$ at $180000 \mathrm{~g}$ ). The wash-step was repeated with membrane storage buffer (buffer B without $2 \mathrm{M}$ $\mathrm{KCl}$ ), after which the pellet was resuspended in a small volume (usually $400 \mu \mathrm{l}$ pellet per litre of original culture). This membrane vesicle fraction was frozen in liquid nitrogen and stored at $-80^{\circ} \mathrm{C}$ in small aliquots. The protein concentration in these samples was estimated using the Bradford assay (Bio$\mathrm{Rad}$ ) with BSA as a standard and UhpB enrichment was confirmed and quantified by Western blotting, using a polyclonal antiserum raised against $\mathrm{His}_{6}-\mathrm{UhpB}^{\prime}$.

In vitro phosphorylation reactions. To assay autophosphorylation activity in membranes in which UhpBC was either enriched or absent, samples were incubated with $\left[\gamma_{-}{ }^{32} \mathrm{P}\right] \mathrm{ATP}$ (ICN; stock $7000 \mathrm{Ci} \mathrm{mmol}{ }^{-1} ; 259 \mathrm{TBq} \mathrm{mmol}^{-1}$ ) in a reaction mixture containing $50 \mathrm{mM}$ Tris $/ \mathrm{HCl} \mathrm{pH} \mathrm{8.0,} 5 \mathrm{mM} \mathrm{MgCl}$, $50 \mathrm{mM} \mathrm{KCl}, 0.5 \mathrm{mM}$ EDTA, $1 \mathrm{mM}$ DTT, $10 \%$ (v/v) glycerol (and G6P). Before initiating the reaction with ATP (usually 50 or $100 \mu \mathrm{M}, 10-25 \mathrm{Ci} \mathrm{mmol}^{-1}$ ), the vesicles (with an ISO membrane orientation: Futai \& Tanaka, 1975) were made permeable for G6P (Boehringer Mannheim) by freezing the reaction mixture in liquid nitrogen, followed by thawing on ice.

To assay phosphoryl transfer to UhpA, $\mathrm{His}_{6}$-UhpA was added to the autophosphorylation mixture (now at $\mathrm{pH} 7 \cdot 5$ ) after 2-3 min incubation with $\left[\gamma_{-}{ }^{32} \mathrm{P}\right]$ ATP.

To assay phosphatase activity (in UhpBC-enriched membranes) towards $\mathrm{P} \sim \mathrm{UhpA}, \mathrm{His}_{6}$-UhpA was first phosphorylated using acetyl phosphate. Acetyl $\left[{ }^{32} \mathrm{P}\right]$ phosphate was enzymically synthesized in AcP buffer (25 mM HEPES; $60 \mathrm{mM}$ potassium acetate; $\left.10 \mathrm{mM} \mathrm{MgCl}_{2} ; \mathrm{pH} 7 \cdot 6\right)$ with $50 \mu \mathrm{Ci}[\gamma$ $\left.{ }^{32} \mathrm{P}\right] \mathrm{ATP}$ per unit of $\mathrm{E}$. coli acetate kinase (Boehringer Mannheim), for $30 \mathrm{~min}$ at room temperature. Acetate kinase was removed from the acetyl- $\left[{ }^{32} \mathrm{P}\right]$ phosphate-containing mixture by filtration through a Centricon $(10 \mathrm{kDa}$ cut-off filter) microconcentrator (Amicon), including extensive filter rinsing in AcP buffer. His $_{6}$-UhpA $(5-15 \mu \mathrm{M})$ and acetyl $\left[{ }^{32} \mathrm{P}\right]$ phosphate were mixed, and incubated for $2.5 \mathrm{~h}$ at $30^{\circ} \mathrm{C}$. Similarly, nonradioactive $\mathrm{P} \sim$ UhpA was obtained by incubating $5-15 \mu \mathrm{M}$ $\mathrm{His}_{6}$-UhpA with $10 \mathrm{mM}$ acetyl phosphate and $10 \mathrm{mM} \mathrm{MgCl}$ in TEDG buffer $(50 \mathrm{mM}$ Tris/HCl pH 7.5; $1 \mathrm{mM}$ DTT; $0.5 \mathrm{mM}$ EDTA; $10 \%, \mathrm{v} / \mathrm{v}$, glycerol), at $30{ }^{\circ} \mathrm{C}$ for $2.5 \mathrm{~h}$. In both cases, P $\sim$ UhpA (free of remaining acetyl phosphate, ATP and/or ADP) was collected by exchanging the buffer via a BioGel P-6 microcolumn equilibrated in TEDG buffer. The actual phosphatase reaction was initiated by adding (G6P-containing) membrane vesicles to the $\mathrm{P} \sim$ UhpA product in $50 \mathrm{mM}$ Tris/ $\mathrm{HCl} \mathrm{pH} \mathrm{7.5,} 5 \mathrm{mM} \mathrm{MgCl}_{2}, 50 \mathrm{mM} \mathrm{KCl}, 0 \cdot 5$ mM EDTA, $1 \mathrm{mM} \mathrm{DTT}$ and $10 \%$ (v/v) glycerol.

The (de)phosphorylation reactions, carried out at room temperature, were quenched by adding $3 \times$ SDS-loading buffer (containing $30 \mathrm{mM}$ EDTA), after which the samples were kept on ice. Before loading they were heated for $3 \mathrm{~min}$ at $55^{\circ} \mathrm{C}$ and then separated by SDS-PAGE. Gels were first washed in $25 \mathrm{mM}$ phosphate buffer $(\mathrm{pH} 7 \cdot 5)$, then in demineralized water and subsequently dried under vacuum. Phosphorylated proteins were visualized and quantified by autoradiography (Kodak X-Omat AR film) and/or phosphorimaging (Bio$\mathrm{Rad})$. The experiments described were performed at least three independent times with a reproducible outcome; typical examples are shown in the results.

Immunodetection. For Western blotting, total cellular protein, membrane-protein fractions, and purified $\mathrm{His}_{6}-\mathrm{UhpB}^{\prime}$ or $\mathrm{His}_{6}$-UhpA (5-100 ng) were separated by SDS-PAGE and then transferred to $0.4 \mu \mathrm{m}$ nitrocellulose membranes using a semidry blotting system (Bio-Rad). Membranes were treated with a polyclonal rabbit antiserum (Institute made) against

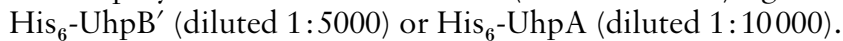
Antisera were raised in New Zealand White rabbits (Institute facility). Horseradish-peroxidase-conjugated goat anti-rabbit IgG (Bio-Rad) was used as secondary antibody, followed by treatment with SuperSignal chemiluminescent substrate (Pierce). The secondary antibody signal was detected and quantified by exposure of the membranes to film (Kodak XOmat AR), followed by densitometric analysis.

\section{RESULTS}

\section{G6P-induced autophosphorylation of UhpB}

As activation of the Uhp system in vivo is known to be dependent on the presence of extracellular G6P, we initiated experiments to demonstrate the supposed G6Pdependent UhpB autophosphorylation in an in vitro system. Preliminary experiments revealed that this would be extremely difficult in membranes derived from wild-type strains; therefore, UhpBC-enriched membranes were used. Membranes from a $u h p B C$ deletion strain served as a control. Furthermore, since a French press was used to generate the membrane fragments, their orientation was predominantly ISO (Futai \& Tanaka, 1975). Consequently, the presumed binding site of UhpC for G6P is located on the inside of these membrane vesicles. A major obstacle in this experimental design is the intra-vesicular localization of the G6P binding site. We allowed G6P access to the intravesicular compartment in three alternative ways: (i) via French pressing the cells in the presence of G6P; (ii) via quickly freezing the vesicles in liquid nitrogen in the presence of G6P and slowly thawing them on ice; and (iii) via gentle pre-treatment with a low concentration of a non-ionic detergent $(0 \cdot 1 \%, \mathrm{v} / \mathrm{v}$, Triton X-100). All three procedures were effective for G6P concentrations above $100 \mu \mathrm{M}$. As can be clearly seen on the autoradiogram in Fig. 1(a), UhpB (with a calculated mass of $48 \mathrm{kDa}$ and migrating at an apparent mass of $\sim 50 \mathrm{kDa}$ ) was clearly phosphorylated in UhpBC-enriched membranes, isolated in the presence of G6P (lane 3). Without G6P addition, only slight (constitutive) UhpB autophosphorylation was observed (lane 2). As expected, this band was completely absent in membranes lacking UhpBC (lane 1). The additional bands in the gels represent unidentified and non-specifically labelled membrane proteins, of which especially X1 was highly competitive with UhpB for the added $\left[\gamma_{-}{ }^{32} \mathrm{P}\right] \mathrm{ATP}$. The time dependence of autophosphorylation of membranebound UhpB is shown in Fig. 1(b). P UhpB formation was linear during the first minute after addition of ATP and reached a maximum after $2 \mathrm{~min}$. Subsequently gradual dephosphorylation occurred. 

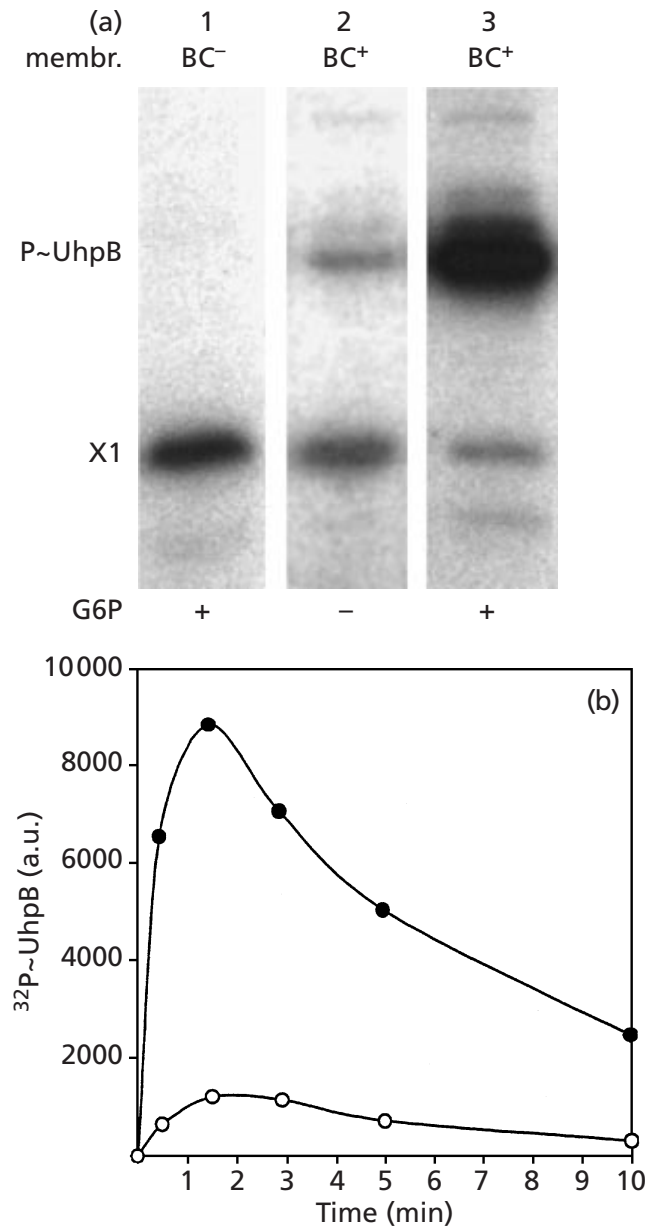

Fig. 1. G6P-induced UhpB autophosphorylation. Reactions were initiated by adding $50 \mu \mathrm{M}$ [ $\left.\gamma_{-}{ }^{32} \mathrm{P}\right]$ ATP to ISO membranes in phosphorylation buffer $\mathrm{pH} 8 \cdot 0$. (a) Samples were quenched after 2 min and separated by SDS-PAGE ( $11 \%$ gels), followed by autoradiography. Lanes: 1, UhpB-lacking membranes, plus $1 \mathrm{mM} \mathrm{G6P}$; 2, UhpB-enriched membranes with no added G6P; 3, UhpB-enriched membranes, plus $1 \mathrm{mM}$ G6P. X1 was an unidentified non-specifically labelled membrane protein which was especially competitive with UhpB for ATP. (b) Time-course of autophosphorylation (in arbitrary units; see Methods) of membrane-bound UhpB $(0.25 \mu \mathrm{M})$ in UhpBC-enriched membranes, in the absence $(O)$ or presence $(0)$ of $0.5 \mathrm{mM} \mathrm{G6P}$.

As an alternative we also tested right-side-out (RSO) membrane vesicles, prepared according to the method of Kaback (1971) and optimized for autophosphorylation assays as described by Jung et al. (2000). These experiments qualitatively confirmed the result obtained with ISO vesicles (data not shown).

We then analysed the concentration range of G6P that stimulates UhpB autophosphorylation in this in vitro assay, to determine the apparent $K_{\mathrm{m}}^{\mathrm{G} 6 \mathrm{P}}$ for transmembrane signalling via UhpBC. Experiments using all three above-mentioned methods to allow G6P access to the intra-vesicular compartment were performed at a range of physiological G6P concentrations $(0-300 \mu \mathrm{M})$. Inclusion of G6P via the freeze-thaw treatment gave the most reproducible results (as determined via Phosphor-

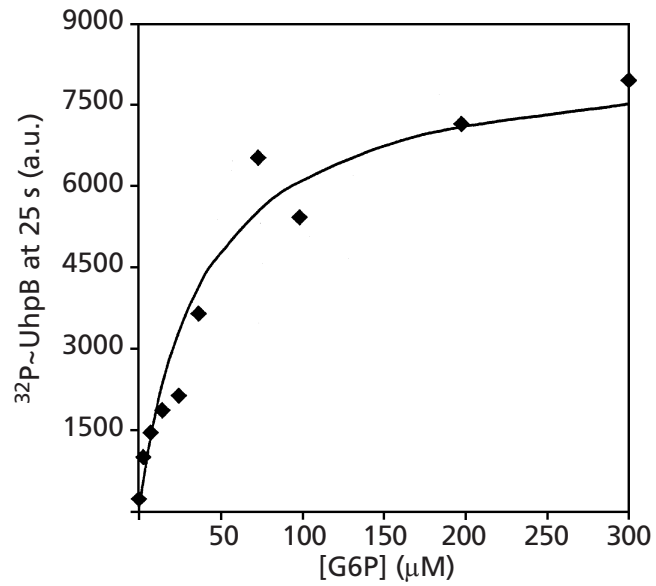

Fig. 2. Initial rate of UhpB autophosphorylation (in arbitrary units) as a function of G6P concentration. G6P was incorporated via freeze-thaw treatment. UhpBC-enriched membranes $(0.15 \mu \mathrm{M}$ UhpB) in phosphorylation buffer $(\mathrm{pH} \mathrm{8.0)}$ were incubated with $250 \mu \mathrm{M}$ ATP and the indicated G6P concentrations. The reactions were initiated by the addition of $\left[\gamma^{-32} P\right] A T P$ and were quenched after $25 \mathrm{~s}$.

Imager pixel quantification of $\mathrm{P} \sim \mathrm{UhpB}$ bands) for the initial rate of $\mathrm{P} \sim \mathrm{UhpB}$ formation. A typical example of such an experiment is shown in Fig. 2, where a high ATP concentration $(250 \mu \mathrm{M})$ was used to assure maximal initial rates. The half-maximal rate of $\mathrm{P} \sim \mathrm{UhpB}$ formation, measured at $40 \mu \mathrm{M}$ in the experiment shown, was observed at $25( \pm 10) \mu \mathrm{M}$ G6P.

To obtain additional kinetic characteristics of membrane-bound UhpB autophosphorylation, the same assay as described above was done, but with varying concentrations of ATP (with equal specific activity), and the initial rate (i.e. within $30 \mathrm{~s}$ of ATP addition) of $\mathrm{P} \sim \mathrm{UhpB}$ formation for each ATP concentration (now with a saturating concentration of G6P) was determined. From these experiments, the $K_{\mathrm{m}}^{\mathrm{ATP}}$ for the initial rate of autophosphorylation of membrane-bound UhpB was estimated to be $\sim 250 \mu \mathrm{M}$.

\section{Phosphorylation and dephosphorylation of UhpA by membrane-bound UhpB}

To investigate phosphoryl transfer from UhpB to UhpA, membrane-bound UhpB was first incubated with $[\gamma$ $\left.{ }^{32} \mathrm{P}\right]$ ATP to achieve maximum autophosphorylation of the kinase (Fig. 3a, lane 1). Subsequently, purified $\mathrm{His}_{6}$ UhpA was added. In Fig. 3(a) this is shown for a UhpB: UhpA molar ratio of 1:1 (lane 2). The phosphoryl transfer reaction was quenched after $30 \mathrm{~s}$. Within this time interval $60 \%$ of the label detected in UhpB was released as inorganic phosphate and $25 \%$ reappeared as ${ }^{32} \mathrm{P} \sim \mathrm{UhpA}$. The underlying time-course of UhpA phosphorylation was further analysed for different UhpB:UhpA molar ratios. $\mathrm{P} \sim \mathrm{UhpA}$ formation appeared to be rapid and transient (Fig. 3b), with an optimum ratio (i.e. maximum level of UhpA phos- 

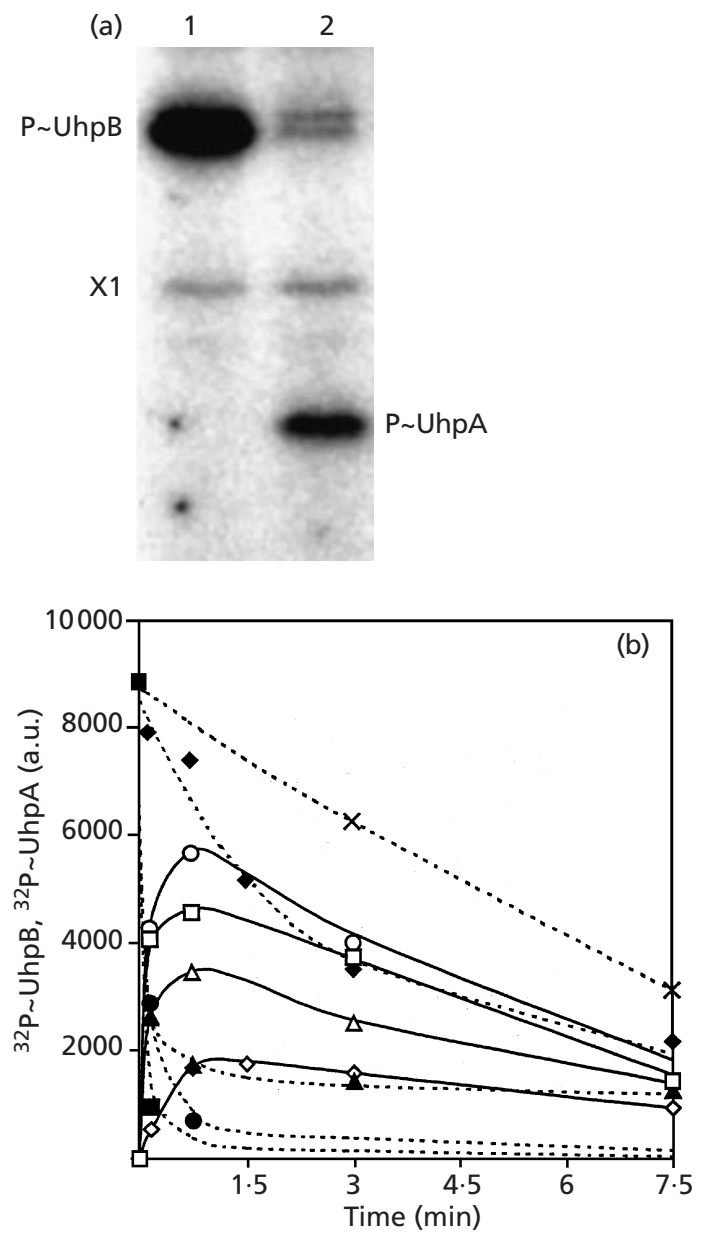

Fig. 3. Phosphoryl transfer from membrane-bound $P \sim$ UhpB to $\mathrm{His}_{6}$-UhpA. (a) UhpB autophosphorylation in UhpBC-enriched membranes, containing $1 \mu \mathrm{M}$ membrane-bound $\mathrm{UhpB}$ and initiated with $50 \mu \mathrm{M}$ [ $\left.\gamma_{-}{ }^{32} \mathrm{P}\right] \mathrm{ATP}$ in phosphorylation buffer $\mathrm{pH} 7 \cdot 5$, was either quenched after 2 min (lane 1) or $1 \mu \mathrm{M} \mathrm{His}_{6}-$ UhpA was added after $2 \mathrm{~min}$ and the reaction was quenched after an additional $30 \mathrm{~s}$ (lane 2). (b) Time course of (de)phosphorylation of UhpA (open symbols, regular lines) and UhpB (solid symbols, dotted lines), at increasing UhpA:UhpB ratios. UhpA (final concentration $0,0.125,0.625,1.25$ and $2.5 \mu \mathrm{M}$, respectively) was added to the autophosphorylation mixture $(0.25 \mu \mathrm{M}$ membrane-bound UhpB, initiated with $\left.100 \mu \mathrm{M}\left[\gamma_{-}{ }^{32} \mathrm{P}\right] \mathrm{ATP}\right)$ at $2 \mathrm{~min}$ and samples were quenched after the indicated times. Symbols indicate different UhpA:UhpB ratios: $\times, 0: 1$; squares, $10: 1$; circles, $5: 1$; triangles, $2.5: 1$; diamonds, 0.5:1.

phorylation) of 1:5 (UhpB: UhpA). This relatively low optimal ratio may be due to a very high level of phosphatase activity of UhpB towards $\mathrm{P} \sim \mathrm{UhpA}$. By first phosphorylating UhpA, using acetyl $\left[{ }^{32} \mathrm{P}\right]$ phosphate, this could be further addressed. Due to the enzymic procedure used to synthesize acetyl $\left[{ }^{32} \mathrm{P}\right]$ phosphate, this reaction resulted in phosphorylated UhpA with a high specific activity, but a low chemical concentration $(\sim 0.5 \mu \mathrm{M})$ and an excess of non-phosphorylated UhpA. This may represent the in vivo situation; however, a higher initial $\mathrm{P} \sim \mathrm{UhpA}$ concentration was nevertheless desirable. This was achieved
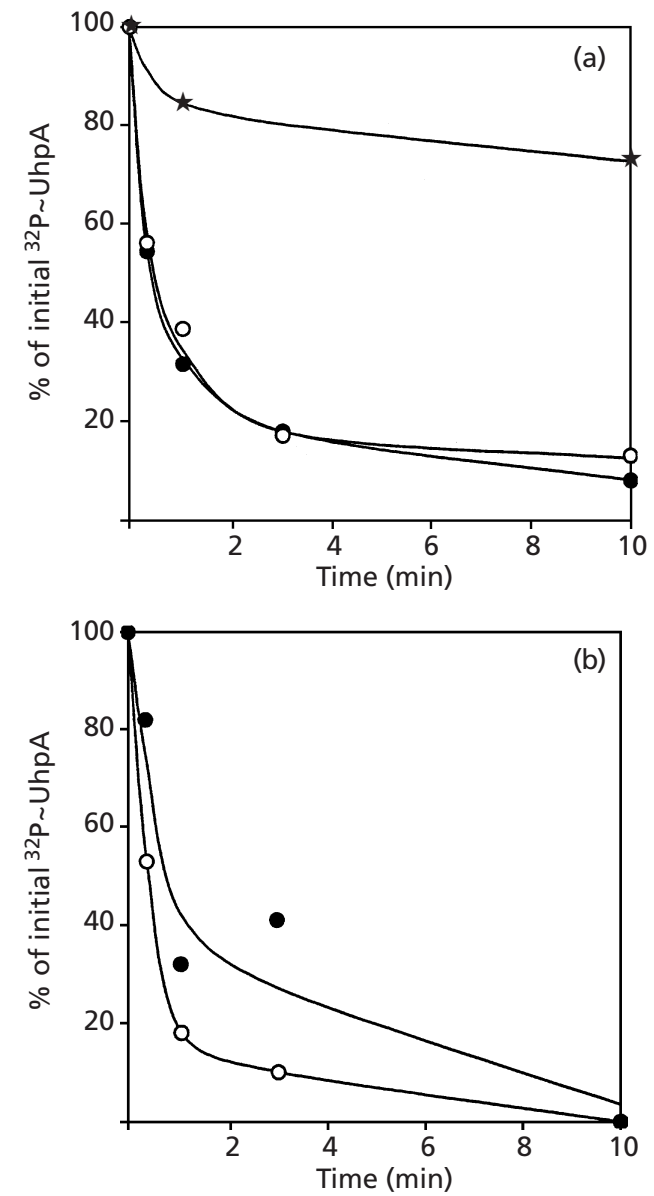

Fig. 4. $\mathrm{P} \sim$ UhpA dephosphorylation by $U$ hpBC-enriched membranes. UhpA $(10 \mu \mathrm{M})$ was phosphorylated using acetyl phosphate, as described in Methods. Membranes, containing either $1 \mu \mathrm{M}(\mathbf{O} / O,+/-1 \mathrm{mM}$ G6P, respectively), or no membrane-bound UhpB $(\star)$, were added to (P ) UhpA at time 0 and samples were taken at the indicated time-points. (a) $<10 \% \mathrm{P} \sim$ UhpA at time 0 ; (b) $>60 \% \mathrm{P} \sim$ UhpA at time 0 .

by synthesizing non-radioactive $\mathrm{P} \sim \mathrm{UhpA}$ separately and combining it with ${ }^{32} \mathrm{P} \sim$ UhpA just prior to initiating the phosphatase assay. The experiment displayed in Fig. $4(\mathrm{a}, \mathrm{b})$ shows that upon addition of UhpBC-enriched membranes, P UhpA was rapidly dephosphorylated, as compared with the addition of membranes without UhpBC. This rapid dephosphorylation occurred independently of the presence or absence of intra-vesicular G6P (compare open and solid symbols) and the absolute concentration of $\mathrm{P} \sim \mathrm{UhpA}$ (compare Fig. $4 \mathrm{a}$ and $4 \mathrm{~b}$ ).

\section{DISCUSSION}

Although the majority of intracellular phosphoryl transfer pathways based on the two-component paradigm use a transmembrane signal transfer step to become activated, most systems sense an environmental condition that is not easily mimicked in an in vitro membrane set-up that allows assay of signal-stimulated HPK autophosphorylation. Fortunately, several bac- 
terial signalling systems with signal input and detection confined to the extracellular presence of a single molecular species have been characterized. In E. coli, direct sensing of a ligand by a sensory HPK (presumably) occurs in NarX, NarQ (Rabin \& Stewart, 1993), PhoQ (Kato et al., 1999), DcuS (Zientz et al., 1998), TorS (Jourlin et al., 1996), CusS, PcoS (Munson et al., 2000) and $\mathrm{HydH}$ (ZraS) (Leonhartsberger et al., 2001). Of these, sensitivity tests using in vitro autophosphorylation assays have been reported for membranebound NarX (Lee et al., 1999; Williams \& Stewart, 1997) and PhoQ (from Salmonella; Castelli et al., 2000; Montagne et al., 2001), as well as for the 'turgor sensor' $\mathrm{KdpD}$, despite the complex nature of the stimulus which activates this system (Jung et al., 2000).

The Uhp system is also activated upon sensing a single extracytoplasmic ligand (G6P). However, the membrane-bound HPK, UhpB, is incapable of showing signal-stimulated autophosphorylation on its own (Island \& Kadner, 1993). An additional membranebound protein, UhpC, is needed to sense G6P and to elicit autophosphorylation in the cytoplasmic domain of UhpB. The existence of this proposed UhpBC signalling complex has been thoroughly tested in vivo by Kadner and colleagues (Island \& Kadner, 1993; Wright et al., 2000). Here we have investigated the phosphorylation characteristics of UhpBC in vitro, using an ISO membrane set-up. We observed that the range of concentrations at which G6P activates signalling (i.e. UhpB autophosphorylation) overlaps with the range of concentrations of G6P that in vivo determine the Uhp output (data not shown; Shattuck-Eidens \& Kadner, 1981). Co-operative effects downstream in the signal transduction pathway may explain the lower overall apparent $K_{\mathrm{m}}^{\mathrm{G} 6 \mathrm{P}}$ for the induction observed in vivo.

The three phosphoryl-transfer reactions that occur within the Uhp signal transduction pathway were addressed here in vitro in a stepwise fashion. The occurrence of all three activities, viz. autophosphorylation (1), phosphoryl transfer (2) and phosphatase activity (3), was demonstrated and characterized independently. This represents the first overall in vitro analysis of phosphoryl flow through the Uhp system and, indeed, the outcome confirms the hypothesized two-component paradigm. However, none of the three assays adequately imitates the in vivo situation, where all three regulatory Uhp proteins are simultaneously present. Under these conditions, sensing of G6P by UhpC induces autophosphorylation in UhpB and $\mathrm{P} \sim$ UhpB instantaneously serves as a substrate for UhpA. The latter is present in a large molar excess over UhpB (data not shown).

Here we have demonstrated that the G6P concentration controls the rate of UhpA phosphorylation via the rate of UhpB phosphorylation. G6P does not directly affect the rate of phosphoryl transfer from $\mathrm{P} \sim \mathrm{UhpB}$ to UhpA, nor the phosphatase activity of UhpB towards $\mathrm{P} \sim$ UhpA. In our phosphoryl transfer assay the default phosphatase state (Wright et al., 2000) of UhpB was only overcome for a few minutes after the addition of ATP, whereas, as demonstrated with the $\mathrm{P} \sim \mathrm{UhpA}$ phosphatase assay, the presence of G6P in UhpBC-enriched vesicles could not switch off UhpB phosphatase activity towards $\mathrm{P} \sim \mathrm{UhpA}$. This 'single regulation' phenomenon, i.e. the UhpB phosphatase activity remains constant while G6P turns on the UhpBC kinase activity, is in agreement with the general model proposed by Ninfa (1996). The dependence of UhpB kinase activity on the ATP concentration implies that in vivo, with cytoplasmic ATP concentrations in the order of 4-5 mM (Rohwer et al., 1996), the UhpBC sensor will be 'kinase on' with respect to UhpA phosphorylation, as long as extracellular G6P is being sensed.

For the EnvZ/OmpR system it has recently been shown that $\mathrm{P} \sim \mathrm{OmpR}$ is stabilized when bound to its own target promoter DNA (Qin et al., 2001). This followed a study that demonstrated enhancement of OmpR phosphorylation when it was bound to promoter DNA (Ames et al., 1999). In the former study, $\mathrm{P} \sim \mathrm{OmpR}-\mathrm{DNA}$ sequestration appeared to inhibit phosphatase activity by EnvZ [via squelching of $(\mathrm{P} \sim) \mathrm{OmpR}]$. The possibility of squelching/sequestration of UhpA and OmpR by the phosphoryl transfer domain of UhpB and EnvZ, respectively, has recently been described in detail (Wright \& Kadner, 2001; Wright et al., 2000). However, the 'P UhpA protecting' role of $u h p T$ promoter DNA in the presence of UhpB has not yet been addressed. Moreover, the in vivo relevance of these reported phenomena in wild-type cells remains to be clarified, taking into account that both systems have in common that their RRs exist in large molar excess relative to their cognate HPKs $(\sim 100: 1)$, and that there are only a limited number of RR target promoters on the chromosome. Furthermore, this HPK:RR molar ratio, as well as the demonstration of HPK-RR squelching inhibition, makes enhanced phosphorylation in a pre-existing HPK-RR complex, as very recently reported for FixL-FixJ (Tuckerman et al., 2001), unlikely for the Uhp system, when taking into account that for the latter system the HPK and RR are present in equimolar concentrations (Tuckerman et al., 2001).

Finally, our quantification of the in vivo concentration of UhpA $(2-3 \mu \mathrm{M}$; unpublished data), in combination with the recently reported affinity of $(\mathrm{P} \sim) \mathrm{UhpA}$ for the promoter region of $u h p T$ (Chen \& Kadner, 2000; Olekhnovich et al., 1999), implies that the $u h p T$ promoter region is already saturated with UhpA even in the absence of extracellular G6P. However, P UhpA has a much higher affinity for the $u h p T$ promoter region (Chen \& Kadner, 2000). Therefore, in G6P-induced cells phosphorylation of a relatively small fraction of the total amount of UhpA may well be sufficient to induce uhp $T$ transcription.

\section{ACKNOWLEDGEMENTS}

We thank R. J. Kadner for making E. coli strains and pRK18 available. R. Cordfunke, M. Hendrix and I. NugterenRoodzant are gratefully acknowledged for their expert technical assistance. 
This work was supported by the Netherlands Organization for Scientific Research (NWO), through the division for Earth and Life Sciences (Gebied ALW).

\section{REFERENCES}

Ames, S. K., Frankema, N. \& Kenney, L. J. (1999). C-terminal DNA binding stimulates N-terminal phosphorylation of the outer membrane protein regulator OmpR from Escherichia coli. Proc Natl Acad Sci US A 96, 11792-11797.

Castelli, M. E., Garcia Vescovi, E. \& Soncini, F. C. (2000). The phosphatase activity is the target for $\mathrm{Mg}^{2+}$ regulation of the sensor protein PhoQ in Salmonella. J Biol Chem 275, 22948-22954.

Chen, Q. \& Kadner, R. J. (2000). Effect of altered spacing between $u h p T$ promoter elements on transcription activation. J Bacteriol 182, 4430-4436.

Dahl, J. L., Wei, B. Y. \& Kadner, R. J. (1997). Protein phosphorylation affects binding of the Escherichia coli transcription activator UhpA to the $u h p T$ promoter. J Biol Chem 272, 1910-1919.

Dietz, G. W., Jr (1976). The hexose phosphate transport system of Escherichia coli. Adv Enzymol Relat Areas Mol Biol 44, 237-259.

Dutta, R., Qin, L. \& Inouye, M. (1999). Histidine kinases : diversity of domain organization. Mol Microbiol 34, 633-640.

Futai, M. \& Tanaka, Y. (1975). Localization of D-lactate dehydrogenase in membrane vesicles prepared by using a french press or ethylenediaminetetraacetate-lysozyme from Escherichia coli. J Bacteriol 124, 470-475.

Goldenbaum, P. E. \& Farmer, K. S. (1980). uhp-Directed, glucose 6-phosphate membrane receptor in Escherichia coli. J Bacteriol 142, 347-349.

Grebe, T. W. \& Stock, J. B. (1999). The histidine protein kinase superfamily. Adv Microb Physiol 41, 139-227.

Island, M. D. \& Kadner, R. J. (1993). Interplay between the membrane-associated $\mathrm{UhpB}$ and UhpC regulatory proteins. $J$ Bacteriol 175, 5028-5034.

Island, M. D., Wei, B. Y. \& Kadner, R. J. (1992). Structure and function of the $u h p$ genes for the sugar phosphate transport system in Escherichia coli and Salmonella typhimurium. J Bacteriol 174, 2754-2762.

Jourlin, C., Bengrine, A., Chippaux, M. \& Mejean, V. (1996). An unorthodox sensor protein (TorS) mediates the induction of the tor structural genes in response to trimethylamine $\mathrm{N}$-oxide in Escherichia coli. Mol Microbiol 20, 1297-1306.

Jung, K., Veen, M. \& Altendorf, K. (2000). $\mathrm{K}^{+}$and ionic strength directly influence the autophosphorylation activity of the putative turgor sensor KdpD of Escherichia coli. J Biol Chem 275, 40142-40147.

Kaback, H. R. (1971). Bacterial membranes. Methods Enzymol 22, 99-115.

Kadner, R. J. (1995). Expression of the sugar-phosphate transport system of Escherichia coli. In Two-Component Signal Transduction, pp. 263-274. Edited by J. A. Hoch \& T. J. Silhavy. Washington, DC: American Society for Microbiology.

Kato, A., Tanabe, H. \& Utsumi, R. (1999). Molecular characterization of the PhoP-PhoQ two-component system in Escherichia coli K-12: identification of extracellular $\mathrm{Mg}^{2+}$-responsive promoters. J Bacteriol 181, 5516-5520.

Kim, D.-j. \& Forst, S. (2001). Genomic analysis of the histidine kinase family in bacteria and archaea. Microbiology 147, $1197-1212$.
Lee, A. I., Delgado, A. \& Gunsalus, R. P. (1999). Signal-dependent phosphorylation of the membrane-bound NarX two-component sensor-transmitter protein of Escherichia coli: nitrate elicits a superior anion ligand response compared to nitrite. J Bacteriol 181, 5309-5316.

Leonhartsberger, S., Huber, A., Lottspeich, F. \& Bock, A. (2001). The hydH/G genes from Escherichia coli code for a zinc and lead responsive two-component regulatory system. J Mol Biol 307, 93-105.

Merkel, T. J., Nelson, D. M., Brauer, C. L. \& Kadner, R. J. (1992). Promoter elements required for positive control of transcription of the Escherichia coli uhpT gene. J Bacteriol 174, 2763-2770.

Mizuno, T. (1997). Compilation of all genes encoding twocomponent phosphotransfer signal transducers in the genome of Escherichia coli. DNA Res 4, 161-168.

Montagne, M., Martel, A. \& Le Moual, H. (2001). Characterization of the catalytic activities of the PhoQ histidine protein kinase of Salmonella enterica serovar typhimurium. J Bacteriol 183, 1787-1791.

Munson, G. P., Lam, D. L., Outten, F. W. \& O'Halloran, T. V. (2000). Identification of a copper-responsive two-component system on the chromosome of Escherichia coli K-12. J Bacteriol 182, 5864-5871.

Nierman, W. C., Feldblyum, T. V. \& Laub, M. T. \& 34 other authors (2001). Complete genome sequence of Caulobacter crescentus. Proc Natl Acad Sci US A 98, 4136-4141.

Ninfa, A. J. (1996). Regulation of gene transcription by extracellular stimuli. In Escherichia coli and Salmonella typhimurium: Cellular and Molecular Biology, pp. 1246-1261. Edited by F. C. Neidhart and others. Washington, DC: American Society for Microbiology.

Ninfa, E. G., Atkinson, M. R., Kamberov, E. S. \& Ninfa, A. J. (1993). Mechanism of autophosphorylation of Escherichia coli nitrogen regulator II (NRII or NtrB): trans-phosphorylation between subunits. J Bacteriol 175, 7024-7032.

Olekhnovich, I. N., Dahl, J. L. \& Kadner, R. J. (1999). Separate contributions of UhpA and CAP to activation of transcription of the $u h p T$ promoter of Escherichia coli. J Mol Biol 292, 973-986.

Parkinson, J. S. \& Kofoid, E. C. (1992). Communication modules in bacterial signaling proteins. Annu Rev Genet 26, 71-112.

Perraud, A. L., Weiss, V. \& Gross, R. (1999). Signalling pathways in two-component phosphorelay systems. Trends Microbiol 7, 115-120.

Qin, L., Yoshida, T. \& Inouye, M. (2001). The critical role of DNA in the equilibrium between OmpR and phosphorylated OmpR mediated by EnvZ in Escherichia coli. Proc Natl Acad Sci U S A 98, 908-913.

Rabin, R. S. \& Stewart, V. (1993). Dual response regulators (NarL and $\mathrm{NarP}$ ) interact with dual sensors (NarX and NarQ) to control nitrate- and nitrite-regulated gene expression in Escherichia coli K-12. J Bacteriol 175, 3259-3268.

Rodrigue, A., Quentin, Y., Lazdunski, A., Mejean, V. \& Foglino, M. (2000). Two-component systems in Pseudomonas aeruginosa: why so many? Trends Microbiol 8, 498-504.

Rohwer, J. M., Jensen, P. R., Shinohara, Y., Postma, P. W. \& Westerhoff, H. V. (1996). Changes in the cellular energy state affect the activity of the bacterial phosphotransferase system. Eur J Biochem 235, 225-230.

Ronson, C. W., Nixon, B. T. \& Ausubel, F. M. (1987). Conserved domains in bacterial regulatory proteins that respond to environmental stimuli. Cell 49, 579-581.

Sambrook, J., Fritsch, E. F. \& Maniatis, T. (1989). Molecular 
Cloning: a Laboratory Manual, 2nd edn. Cold Spring Harbor, NY: Cold Spring Harbor Laboratory.

Shattuck-Eidens, D. M. \& Kadner, R. J. (1981). Exogenous induction of the Escherichia coli hexose phosphate transport system defined by uhp-lac operon fusions. J Bacteriol 148, 203-209.

Stock, J. B., Ninfa, A. J. \& Stock, A. M. (1989). Protein phosphorylation and regulation of adaptive responses in bacteria. Microbiol Rev 53, 450-490.

Tuckerman, J. R., Gonzalez, G. \& Gilles-Gonzalez, M. A. (2001). Complexation precedes phosphorylation for two-component regulatory system FixL/FixJ of Sinorhizobium meliloti. J Mol Biol 308, 449-455.

Weston, L. A. \& Kadner, R. J. (1988). Role of $u$ hp genes in expression of the Escherichia coli sugar-phosphate transport system. J Bacteriol 170, 3375-3383.

Williams, S. B. \& Stewart, V. (1997). Discrimination between structurally related ligands nitrate and nitrite controls autokinase activity of the NarX transmembrane signal transducer of Escherichia coli K-12. Mol Microbiol 26, 911-925.

Williams, S. B. \& Stewart, V. (1999). Functional similarities among two-component sensors and methyl-accepting chemotaxis proteins suggest a role for linker region amphipathic helices in transmembrane signal transduction. Mol Microbiol 33, 1093-1102.

Winkler, H. H. (1970). Compartmentation in the induction of the hexose-6-phosphate transport system of Escherichia coli. J Bacteriol 101, 470-475.

Wright, J. S. \& Kadner, R. J. (2001). The phosphoryl transfer domain of UhpB interacts with the response regulator UhpA. $J$ Bacteriol 183, 3149-3159.

Wright, J. S., Olekhnovich, I. N., Touchie, G. \& Kadner, R. J. (2000). The histidine kinase domain of UhpB inhibits UhpA action at the Escherichia coli uhpT promoter. J Bacteriol 182, 6279-6286.

Zientz, E., Bongaerts, J. \& Unden, G. (1998). Fumarate regulation of gene expression in Escherichia coli by the DcuSR ( $d c u S R$ genes) two-component regulatory system. J Bacteriol 180, 5421-5425.

Zimmann, P., Puppe, W. \& Altendorf, K. (1995). Membrane topology analysis of the sensor kinase $\mathrm{KdpD}$ of Escherichia coli. J Biol Chem 270, 28282-28288.

Received 13 July 2001; revised 27 July 2001; accepted 22 August 2001. 